\title{
GRAY-SCALE IMAGE ENHANCEMENT USING THE SMQT
}

\author{
Mikael Nilsson, Mattias Dahl, and Ingvar Claesson
}

\author{
Blekinge Institute of Technology \\ School of Engineering \\ Box 520, SE-372 25 Ronneby, Sweden \\ E-mail:mkn@bth.se,mdh@bth.se,icl@bth.se
}

\begin{abstract}
This paper explores the Successive Mean Quantization Transform (SMQT) for automatic enhancement of gray-scale images. The transform is in the paper presented using set theory. The image enhancement capabilities and properties of the transform are analyzed. The transform is capable to perform both a nonlinear and a shape preserving stretch of the image histogram. Experiments and comparisons to histogram equalization are conducted.
\end{abstract}

\section{INTRODUCTION}

Transforming a digital image to a visual more informative new image is a desired goal in several applications. Such transformation is known as image enhancement and could be a way to aid human or machine interpretation. For example producing digital images that render contrast and detail well is a strong requirement in several areas, such as remote sensing, biomedical image analysis and fault detection $[1,2]$. Performing the task automatically without human intervention is particularly hard in image processing. Different approaches and techniques have been suggested to solve this problem [1]-[6]. One well established method is the histogram equalization [3]. Histogram equalization automatically flattens and stretches the dynamic range of the histogram of the image. Hence, an enhancement of the contrast in the image is achieved.

The Successive Mean Quantization Transform (SMQT) has properties that reveal the underlying structure in data [7]. The transform performs an automatic structural breakdown of information. This can be interpreted as a progressive focus on details in an image. These characteristics make the transform interesting for automatic enhancement of any image.

In this paper the SMQT is applied and examined for automatic image enhancement. The transform is presented using set theory. An adjustment parameter is introduced to further control the enhancement. The nonlinear properties of the transform are investigated by means of the histogram change. The image enhancement results are compared to histogram equalization.

\section{DESCRIPTION OF THE SMQT}

Let $x$ be a pixel and $\mathcal{D}(x)$ be a set of $|\mathcal{D}(x)|=D$ pixels. The intensity of a pixel will be denoted $\mathbf{V}(x)$. The SMQT has only one parameter input, the level $L$ (indirectly it will also have the number of pixels $D$ as an important input). The output pixel set from the transform is denoted $\mathcal{M}(x)$. The transform of level $L$ from $\mathcal{D}(x)$ to $\mathcal{M}(x)$ is denoted

$$
\mathrm{SMQT}_{L}: \mathcal{D}(x) \rightarrow \mathcal{M}(x)
$$

The $\mathrm{SMQT}_{L}$ function can be described by a binary tree where the vertices are Mean Quantization Units (MQUs). A MQU consists of three steps, a mean calculation, a quantization and a split of the input set.

The first step of the MQU finds the mean value of the pixels, denoted $\overline{\mathbf{V}}(x)$, according to

$$
\overline{\mathbf{V}}(x)=\frac{1}{|\mathcal{D}|} \sum_{x \in \mathcal{D}} \mathbf{V}(x)
$$

The second step uses the mean to quantize the values of pixels into $\{0,1\}$. Let a comparison function be defined as

$$
\xi(\mathbf{V}(y), \overline{\mathbf{V}}(x))= \begin{cases}1, & \text { if } \mathbf{V}(y)>\overline{\mathbf{V}}(x) \\ 0, & \text { else }\end{cases}
$$

where $y \in \mathcal{D}$. Further, let $\bigotimes$ denote concatenation, and then

$$
\mathcal{U}(x)=\bigotimes_{y \in \mathcal{D}} \xi(\mathbf{V}(y), \overline{\mathbf{V}}(x))
$$

is the mean quantized pixel set. The set $\mathcal{U}(x)$ is the main output from a MQU. The third step splits the input set into two subsets 


$$
\begin{aligned}
& \mathcal{D}_{0}(x)=\{x \mid \mathbf{V}(x) \leq \overline{\mathbf{V}}(x), \forall x \in \mathcal{D}\} \\
& \mathcal{D}_{1}(x)=\{x \mid \mathbf{V}(x)>\overline{\mathbf{V}}(x), \forall x \in \mathcal{D}\}
\end{aligned}
$$

where $\mathcal{D}_{0}(x)$ propagates left and $\mathcal{D}_{1}(x)$ right in the binary tree, see Fig. 1.

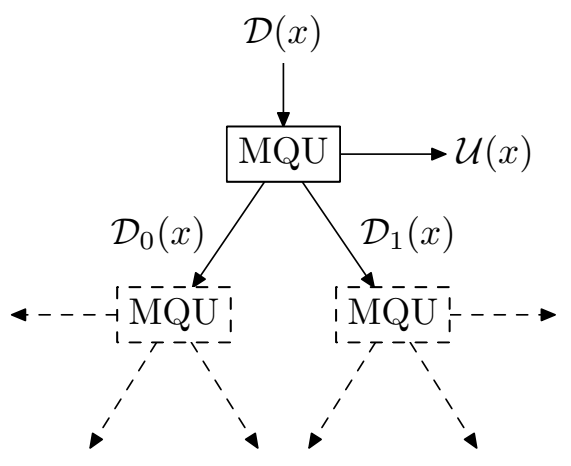

Fig. 1. The operation of one Mean Quantization Unit (MQU).

The output set $\mathcal{U}(x)$ from a MQU is not a value or similarity coefficient as in linear transforms. Instead, $\mathcal{U}(x)$ can be interpreted as the structure of $\mathcal{D}(x)$. Hence, the MQU is independent due to gain and bias adjustments of the input since the mean will follow accordingly. The MQU constitutes the main computing unit for the SMQT. The first level transform, $\mathrm{SMQT}_{1}$, is based on the output from a single MQU, where $\mathcal{U}$ is the output set at the root node. The outputs in the binary tree need extended notation. Let the output set from one MQU in the tree be denoted $\mathcal{U}_{(l, n)}$ where $l=1,2, \ldots, L$ is the current level and $n=1,2, \ldots, 2^{(l-1)}$ is the output number for the MQU at level $l$, see Fig. 2 . Weighting of the values of the pixels in the $\mathcal{U}_{(l, n)}$ sets are performed and the final $\mathrm{SMQT}_{L}$ is found by adding the results. The weighting is performed by $2^{L-l}$ at each level $l$. Hence, the result for the $\mathrm{SMQT}_{L}$ can be found as

$$
\begin{aligned}
\mathcal{M}(x)= & \left\{x \mid \mathbf{V}(x)=\sum_{l=1}^{L} \sum_{n=1}^{2^{l-1}} \mathbf{V}\left(u_{(l, n)}\right) \cdot 2^{L-l}\right. \\
& \left.\forall x \in \mathcal{M}, \forall u_{(n, l)} \in \mathcal{U}_{(l, n)}\right\}
\end{aligned}
$$

As a consequence of this weighing the number of quantization levels, denoted $Q_{L}$, for a structure of level $L$ will be $Q_{L}=2^{L}$.

\section{GRAY-SCALE IMAGE ENHANCEMENT}

A straight forward way to enhance an image is to use the SMQT directly. The only parameter to adjust is the level

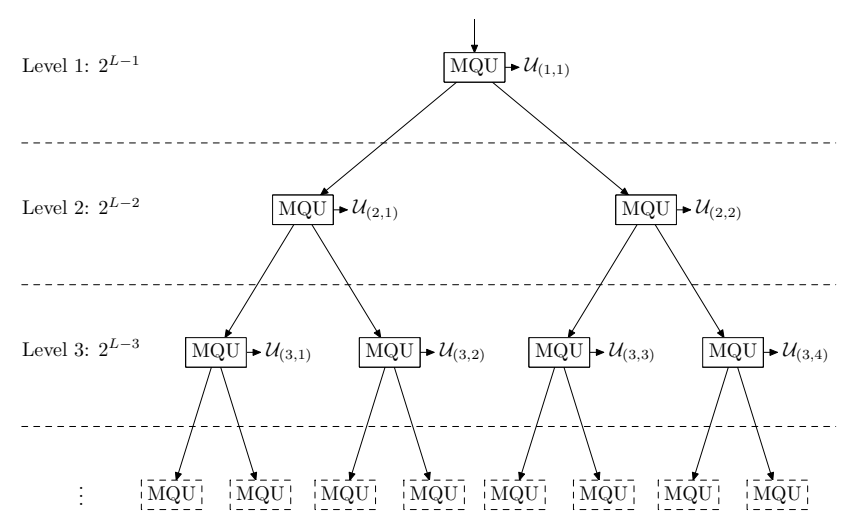

Fig. 2. The Successive Mean Quantization Transform (SMQT) as a binary tree of Mean Quantization Units (MQUs).

$L$. Today digital imaging devices typically use the range $0 \ldots 255$, that is 8 bits is used. For automatic image enhancement of 8 bits images $L$ is chosen to 8 . Nevertheless, it could be convenient to control the amount of enhancement applied. Given the original pixel set $\mathcal{D}(x)$ and the $\mathrm{SMQT}_{8}$ enhanced pixel set $\mathcal{M}(x)$, a simple mix of the two sets can be found as

$$
\begin{aligned}
\mathcal{T}(z)= & \{z \mid \mathbf{V}(z)=(1-\alpha) \mathbf{V}(x)+\alpha \mathbf{V}(y), \\
& \forall x \in \mathcal{D}, \forall y \in \mathcal{M}\}
\end{aligned}
$$

where $\alpha$ is the blending factor. For default enhancement is $\alpha=1$ chosen, for no enhancement is $\alpha=0$ chosen and values in between controls the amount of blending. This type of control could for example be convenient for a human interpreting digital X-rays. However, $\alpha=1$ is the default setting.

Consider the enhancement of the image found in Fig. 3. Note the contrast enhancement achieved by the SMQT. Investigations of the properties for the enhancement can be performed by analyzing the histogram, see Fig. 4 .

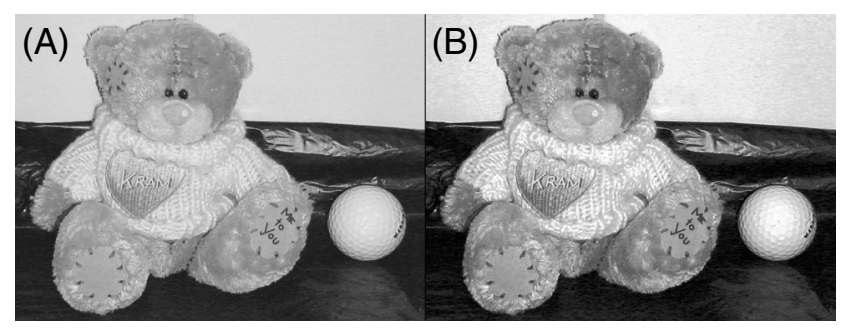

Fig. 3. (A) Original image. (B) $\mathrm{SMQT}_{8}$ enhanced image.

In the original image histogram it is possible to see that this image does not take advantage of the full dynamic range. 

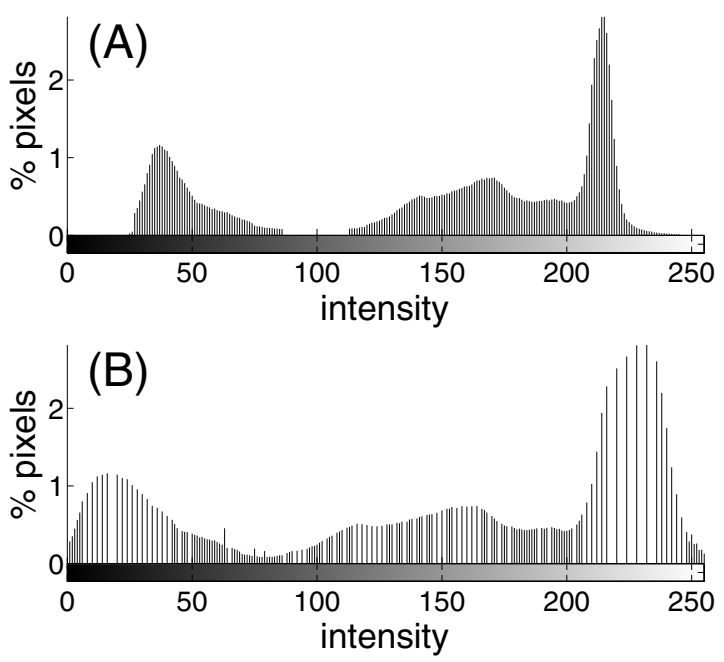

Fig. 4. (A) Original image histogram. (B) $\mathrm{SMQT}_{8}$ enhanced image histogram.

For example intensities between $0-25,90-110$ and $230-$ 255 are not used or poorly explored. The SMQT enhanced image histogram retains the basic shape of the original but stretches it to explore the whole dynamic range. Hence, the SMQT adapts the shape of the histogram by performing a nonlinear stretch. The nonlinear properties of the SMQT will yield a balanced stretch of the histogram. These desirable properties come from the nonlinear successive quantization based on the mean.

\section{EXPERIMENTAL RESULTS}

In order to demonstrate the performance of the SMQT based image enhancement, comparison with histogram equalization is conducted. Note that no parameter tuning is required, that is $L=8$ and $\alpha=1$ are the standard settings for automatic enhancement of 8 bit images. The original image, the blending of the original and the SMQT enhance image, the SMQT enhanced image and the histogram equalized image is presented for ten images, see Figs. 5-6. Please note, that face images from Yale Face Database B [8] have been used to produce the results in Fig. 5.

The histogram equalization has some problems with oversaturation and artifacts in several areas area in the images. Notice how the histogram equalized images have a tendency to get washed out or unnatural. These effects do not occur, or are very limited, in the SMQT enhanced images. This because the SMQT preserves the basic shape of the histogram in contrast to the flattened histogram obtained from histogram equalization.

\section{CONCLUSIONS}

The SMQT has been applied and analyzed for automatic enhancement of images. Properties of the SMQT on images by means of histogram change have been investigated. The SMQT was found to retain the basic shape of the histogram and performs a nonlinear stretch. Hence, the SMQT is found to perform a balanced and natural enhancement of images. A comparison with histogram equalization has been performed, which showed the advantage of the SMQT based enhancement.

\section{REFERENCES}

[1] C. Munteanu and A. Rosa, "Towards automatic image enhancement using genetic algorithms," Proceedings of the 2000 Congress on Evolutionary Computation, vol. 2, pp. 1535-1542, July 2000.

[2] C. Munteanu and A. Rosa, "Gray-scale image enhancement as an automatic process driven by evolution," IEEE Transactions on Systems, Man and Cybernetics, Part B, vol. 34, pp. 1292-1298, April 2004.

[3] William K. Pratt, Digital Image Processing, John Wiley \& Sons, 3rd edition, 2001.

[4] Anil K. Jain, Fundamentals of Digital Image Processing, Prentice-Hall, 1989, ISBN 0-13-336165-9.

[5] Z. Rahman, D.J. Jobson, and G.A. Woodell, "Multiscale retinex for color image enhancement," International Conference on Image Processing, vol. 3, pp. 1003-1006, September 1996.

[6] D.J. Jobson, Z. Rahman, and G.A. Woodell, "Properties and performance of a center/surround retinex," IEEE Transactions on Image Processing, vol. 6, pp. 451-462, March 1997.

[7] M. Nilsson, M. Dahl, and I. Claesson, "The successive mean quantization transform," Accepted for presentation at IEEE International Conference on Acoustics, Speech, and Signal Processing (ICASSP), Philadelphia 2005.

[8] A. S. Georghiades, P. N. Belhumeur, and D. J. Kriegman, "From few to many: Generative models for recognition under variable pose and illumination," in IEEE Int. Conf. on Automatic Face and Gesture Recognition, 2000, pp. 277-284. 


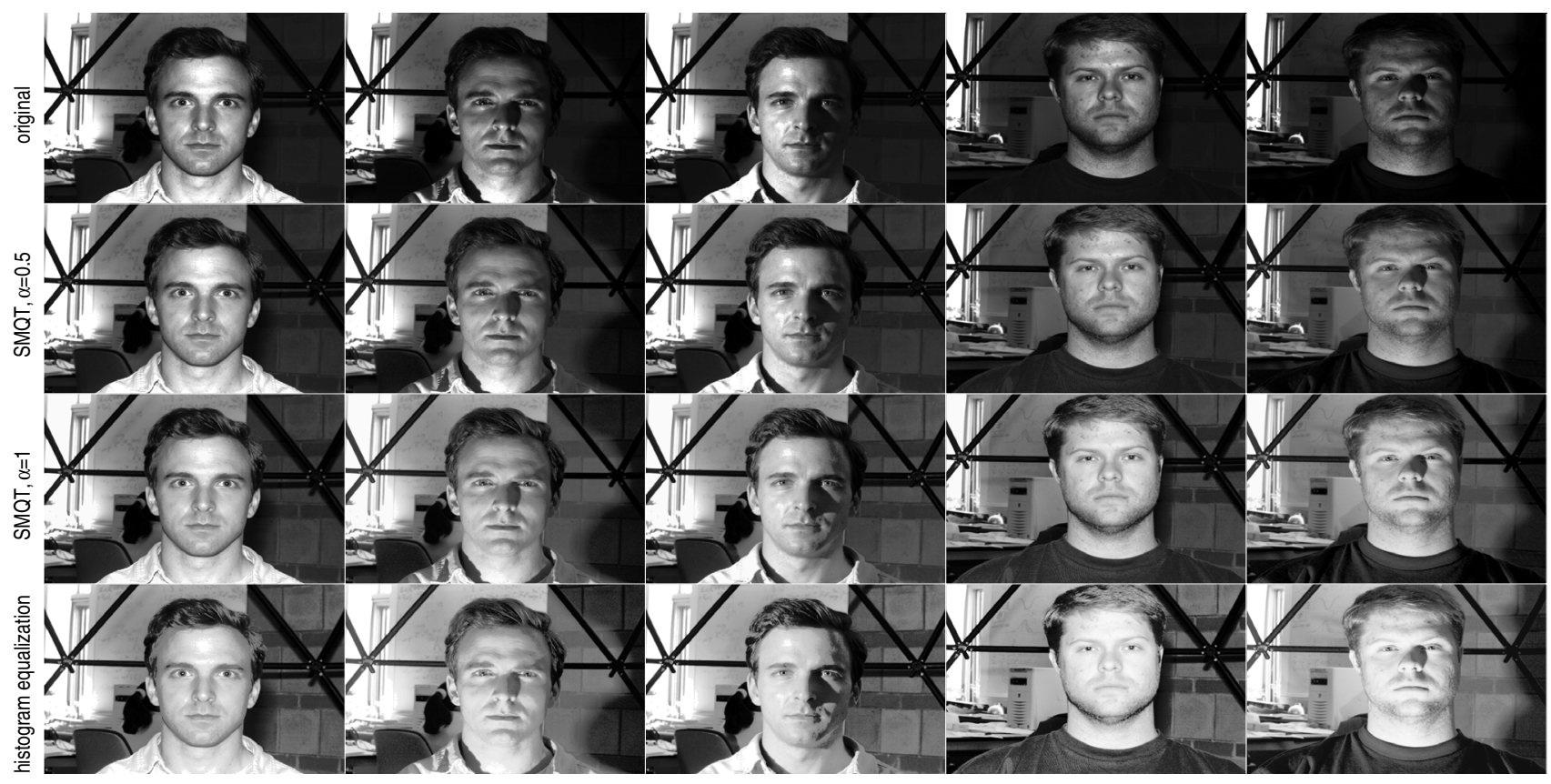

Fig. 5. Image enhancement results, part one. First row original image, second $\mathrm{SMQT}_{8}$ of image blended with original image $(\alpha=0.5)$, third $\mathrm{SMQT}_{8}$ of image $(\alpha=1)$ and fourth histogram equalization of image. Face images are from Yale Face Database B [8]. Zooming in the pdf images is recommended for detail studies. Note the differences of the forehead.
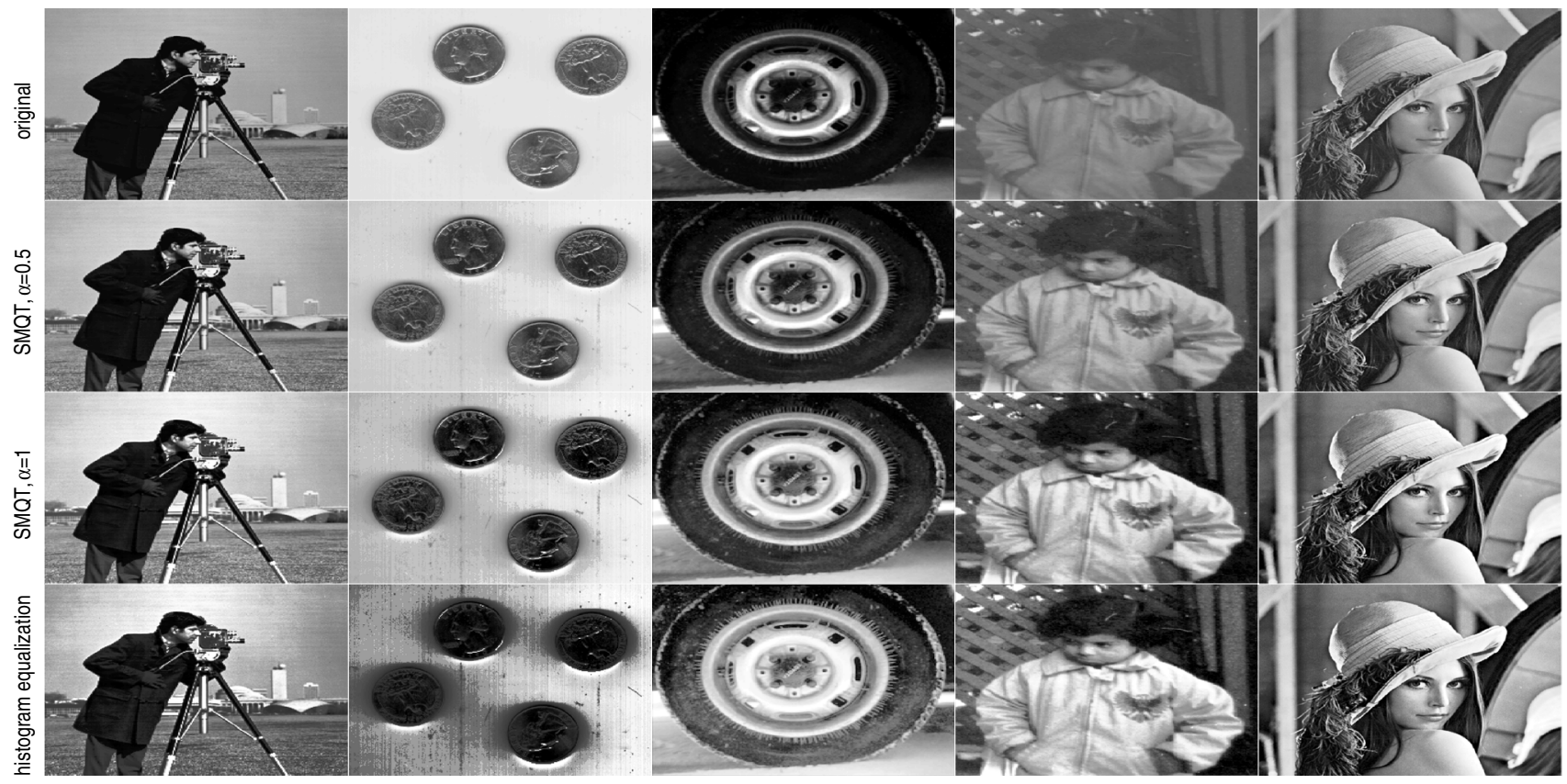

Fig. 6. Image enhancement results, part two. First row original image, second $\mathrm{SMQT}_{8}$ of image blended with original image $(\alpha=0.5)$, third $\mathrm{SMQT}_{8}$ of image $(\alpha=1)$ and fourth histogram equalization of image. Zooming in the pdf images is recommended for detail studies. The enhancement of the image in column four is quite obvious. 